Malaysian Journal of Library \& Information Science, Vol. 10, no.2, Dec 2005: 37-50

\title{
KNOWLEDGE MANAGEMENT: WHY DO WE NEED IT FOR CORPORATES
}

\author{
Bhojaraju G. \\ Librarian-Knowledge Management \\ ICICI OneSource, Bangalore - 560011. India. \\ e-mail: bhojaraju.g@icicionesource.com
}

\begin{abstract}
This article gives a brief introduction about Knowledge Management (KM), its need, definition, components, KM assets, challenges and processes of KM initiative at any organisation. It also provides a narration on how the KM initiative has been adopted at ICICI OneSource, to support the achievement of its Business Process Outsourcing objectives. Both knowledge sharing as well as reuse need to be encouraged and recognized at the individual employee level as well as the company level. This is best done by measuring and rewarding knowledgeperformance. Sustained strategic commitment and a corporate culture that is conducive to knowledge-performance are vital for success in Knowledge Management. The paper concludes with suggestions for the implication for policy and future practices.
\end{abstract}

Keywords: Knowledge management; KM Cyberary; Ontologies; Industrial Credit and Investment Corporation of India Ltd (ICICI)

\section{INTRODUCTION}

Knowledge Management (KM) is the process of gathering, managing and sharing employees' knowledge capital throughout the organisation. Knowledge sharing throughout the organisation enhances existing organisational business processes, introduces more efficient and effective business processes and removes redundant processes. It is a discipline that promotes a collaborative and integrated approach to the creation, capture, organisation access and use of an enterprise's knowledge assets. KM has now become a mainstream priority for companies of all sizes. Capturing a company's most valuable Knowledge (asset) and distributing it 


\section{Bhojaraju G.}

effectively across the enterprise is a business critical issue for many help desk, customer support and IT departments.

The systematic process of finding, selecting, organising, distilling and presenting information, improves an employee's comprehension in a specific area of interest. $\mathrm{KM}$, as emphasized by Abdul Kalam (2004), helps an organisation to gain insight and understanding from its own experience. Specific knowledge management activities help focus on organisation on acquiring, storing and utilizing knowledge for problem solving, dynamic leaning, strategic planning and decision making. It also prevents intellectual assets from decay, adds to firm intelligence and provides increased flexibility.

$\mathrm{KM}$ is not only about Knowledge Technology. KM must be an enabler to achieve strategic business objectives. The organisational debris from failed attempts to impose new technical infrastructures that are either inappropriate to their work environments, or where people are not willing to share knowledge is ample evidence. Hence the need of Knowledge Management initiative arises to become solution for such problems, which brings together people, process and technology and helps corporate to achieve its goals and vision.

Knowledge management is an audit of "intellectual assets" that highlights unique sources, critical functions and potential bottlenecks, which hinder knowledge flows to the point of use. It protects intellectual assets from decay, seeks opportunities to enhance decisions, services and products through adding intelligence, increasing value and providing flexibility. KM complements and enhances other organisational initiatives such as total quality management (TQM), business process re-engineering (BPR) and organisational learning, providing a new and urgent focus to sustain competitive position.

The Gartner Group (2005) defines KM as a discipline that promotes an integrated approach to identifying managing and sharing of all of an enterprise's information assets. These information assets may include database documents, policies procedures as well as previously unarticulated expertise and experience resident in individual workers. Knowledge management issues include developing, implementing and maintaining the appropriate technical and organisational infrastructure to enable knowledge sharing.

Broadbent (1997) defines KM as 'a form of expertise management which draws out tacit knowledge, making it accessible for specific purposes to improve the 
Knowledge Management: Why Do We Need It for Corporates

performance of organisation; about how the organisation's 'know-how' should be structured, organised, located and utilized to provide the most effective action at that point in time'.

\section{WHY DO WE NEED KM FOR CORPORATES}

Knowledge management solutions are now the most important strategic technologies for large companies, according to a new report and survey of European executives by the Economist Intelligence Unit (EIU.com, 2003), sponsored by Tata Consultancy Services. In the survey, 67\% of companies cite knowledge management/business intelligence solutions as important to achieving their strategic goals over the next three years.

To serve customers well and remain in business companies must: reduce their cycle times, operate with minimum fixed assets and overhead (people, inventory and facilities), shorten product development time, improve customer service, empower employees, innovate and deliver high quality products, enhance flexibility and adoption, capture information, create knowledge, share and learn. None of this is possible without a continual focus on the creation, updating, availability, quality and use of knowledge by all employees and teams, at work and in the marketplace.

\section{Components of Knowledge Management}

Based on actual experiences of the leading global KM case studies, the components for KM can be broadly categorized into three classes - People, Processes, and Technology (Figure 1). While all three are critical to build a learning organisation and get business results from $\mathrm{KM}$, a majority of organisations worldwide implementing KM have found it relatively easier to put technology and processes in place, whereas the "people" component has posed greater challenges.

The biggest challenge in KM is to ensure participation by the people or employees in the knowledge sharing, collaboration and re-use to achieve business results. In many organisations, this requires changing traditional mindsets and organisational culture from "knowledge-hoarding" (to keep hidden or private) to "knowledge-sharing" (share among team members) and creating an atmosphere of trust. This is achieved through a combination of motivation / recognition and rewards, re-alignment of performance appraisal systems, and other measurement systems. A key to success in Knowledge Management is to provide people visibility, recognition and credit as 


\section{Bhojaraju G.}

"experts" in their respective areas of specialization - while leveraging their expertise for business success.

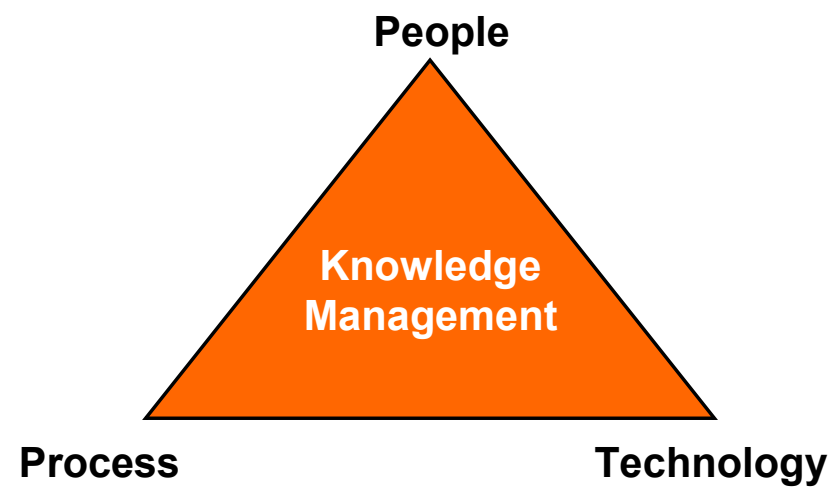

Figure 1: Components of Knowledge Management

The Process component include standard processes for knowledge-contribution, content management (accepting content, maintaining quality, keeping content current, deleting or archiving content that is obsolete), retrieval, membership on communities of practice, implementation-projects based on knowledge-reuse, methodology and standard formats to document best-practices and case studies, etc. It is important for processes to be as clear and simple as possible and wellunderstood by employees across the organisation.

KM technology solutions provide functionality to support knowledge-sharing, collaboration, workflow, document-management across the enterprise and beyond into the extended enterprise. These tools typically provide a secure central space where employees, customers, partners and suppliers can exchange information, share knowledge and guide each other and the organisation to better decisions. The most popular form of KM technology enablement is the Knowledge-Portal on the Corporate Intranet (and extranets where customers, partners and/or suppliers are involved). Common technologies used for knowledge portals include standard Microsoft technologies or Lotus Notes databases. A company must choose a technology option that meets its KM objectives and investment plan. While technology is a key enabler to KM, it is important to ensure that the technology solution does not take the focus away from business issues and is user-friendly and simple to use. Many companies have made the mistake of expending a 


\section{Knowledge Management: Why Do We Need It for Corporates}

disproportionately high portion of their KM effort and resources on technology - at the cost of people-involvement or strategic commitment - resulting in zero or very limited business results. It is also important to remember that users of the KM system are subject-matter experts in their respective areas of specialization and not necessarily IT experts.

\section{KM ASSETS AND PROCESSES}

Typically, there are six knowledge assets in an organisation (Marr, 2003), namely:

1) Stakeholder relationships: includes licensing agreements; partnering agreements, contracts and distribution agreements.

2) Human resources: skills, competence, commitment, motivation and loyalty of employees.

3) Physical infrastructure: office layout and information and communication technology such as databases, e-mail and intranets.

4) Culture: organisational values, employee networking and management philosophy.

5) Practices and routines: formal or informal process manuals with rules and procedures and tacit rules, often refers to "the way things are done around here".

6) Intellectual Property: patents, copyrights, trademarks, brands, registered design and trade secrets.

Knowledge management processes maximize the value of knowledge assets through collaboration, discussions, and knowledge sharing. It also gives value to people's contribution through awards and recognitions. Process includes generation, codification (making tacit knowledge explicit in the form of databases, rules and procedures), application, storing, mapping, sharing and transfer. Together these processes can be used to manage and grow an organisation's intellectual capital.

\section{CHALLENGES FOR MANAGING KNOWLEDGE}

Most often knowledge lies within an organisation implicitly, out of sight, undervalued and underused. Often, it leaves the building when the employees walk out of the company along with them. Managing the flow of knowledge around an organisation, as depicted in Figure 2, is a challenge. The Knowledge management process normally face six challenges at each stage of the process flow (Shadbolt \& 


\section{Bhojaraju G.}

O'Hara, 2003), and failing to meet any of these challenges can derail an organisation's ability to use its knowledge assets to its best advantage. The six challenges are as follows:

a) Knowledge acquisition: The challenge here is to get hold of the information that is around, and turn it into knowledge by making it usable. This might involve, for instance, making tacit knowledge explicit, identifying gaps in the knowledge already held, acquiring and integrating knowledge from multiple sources (e.g. different experts, or distributed sources on the WWW), acquiring knowledge from unstructured media (e.g. natural language or diagrams). Knowledge acquisition (KA) is a field which has reached a certain level of maturity. It began as part of the drive to build knowledge-based systems, and was a line of research devoted to developing methods and software tools to provide knowledge content for such systems.

b) Knowledge Modelling: Modelling bridges the gap between the acquisition of knowledge and its use. Knowledge model structures must be able to represent knowledge so that it can be used for problem-solving. One important knowledge modeling idea is that of ontologies, which are specifications of the generic concepts, attributes, relations and axioms of a knowledge base or domain. Ontologies can act as placeholders and organising structures for acquired knowledge, while also providing a format for understanding how knowledge will be used.

c) Knowledge Retrieval: When a knowledge repository gets very large, finding a particular piece of knowledge can become very difficult. There are two related problems to do with knowledge retrieval. First, there is the issue of finding knowledge again once it has been stored, understanding the structure of your archive in order to navigate through it efficiently. And second, there is the problem of retrieving the subset of content from the repository that is relevant to a particular problem. This second problem, the dynamic extraction of knowledge from a repository, may well set problems for a knowledge retrieval system that alter regularly and quickly during problem-solving.

d) Knowledge Reuse: One of the most serious impediments to cost-effective use of knowledge is that often knowledge bases or systems are constructed afresh. It is unusual for problem-solving experience or domain content to 
Knowledge Management: Why Do We Need It for Corporates

be acquired and then reused, partly because knowledge tends to require different representations depending on the problem-solving that it is intended to do. Understanding the use and application of knowledge would enable more leverage to be gained from the knowledge already at hand, thereby increasing the returns on the investment in those knowledge assets.

e) Knowledge Publishing: The challenge of publishing or disseminating can be described as getting the right knowledge, in the right form, in the right place, to the right person, at the right time. Different users will require knowledge presented and visualized in different ways, and the quality of such presentation is not merely a matter of preference, but can radically affect the value of the knowledge to the user. Getting presentation right will involve understanding the different perspectives of people with different agendas, while an understanding of knowledge content will help to ensure that important related pieces of knowledge get published at the appropriate time.

f) Maintenance: The last challenge is to keep the knowledge repository functional. This may involve the regular updating of content as content changes (e.g. as price lists are revised). But it may also involve a deeper analysis of the knowledge content. Some content has a considerable longevity, while other knowledge dates very quickly. If a repository of knowledge it to remain active over a period of time, it is essential to know which parts of the knowledge base must be discarded and when. Other problems involved in maintenance include verifying and validating the content, and certifying its safety.

\section{KNOWLEDGE MANAGEMENT AT ICICI ONESOURCE}

ICICI stands for "Industrial Credit and Investment Corporation of India Ltd". ICICI OneSource is among India's leading end-to-end Business Process Outsourcing (BPO) service providers with proven full service expertise for consumer industries including financial services, retail, telecom and utilities. IOneSource Limited is majority owned by the ICICI Group (ICICI Onesource, 2005). Its flagship, ICICI Bank, is the largest private sector bank in India. Other stakeholders include Temasek Holdings of Singapore and Westbridge Capital Partners. 


\section{Bhojaraju G.}

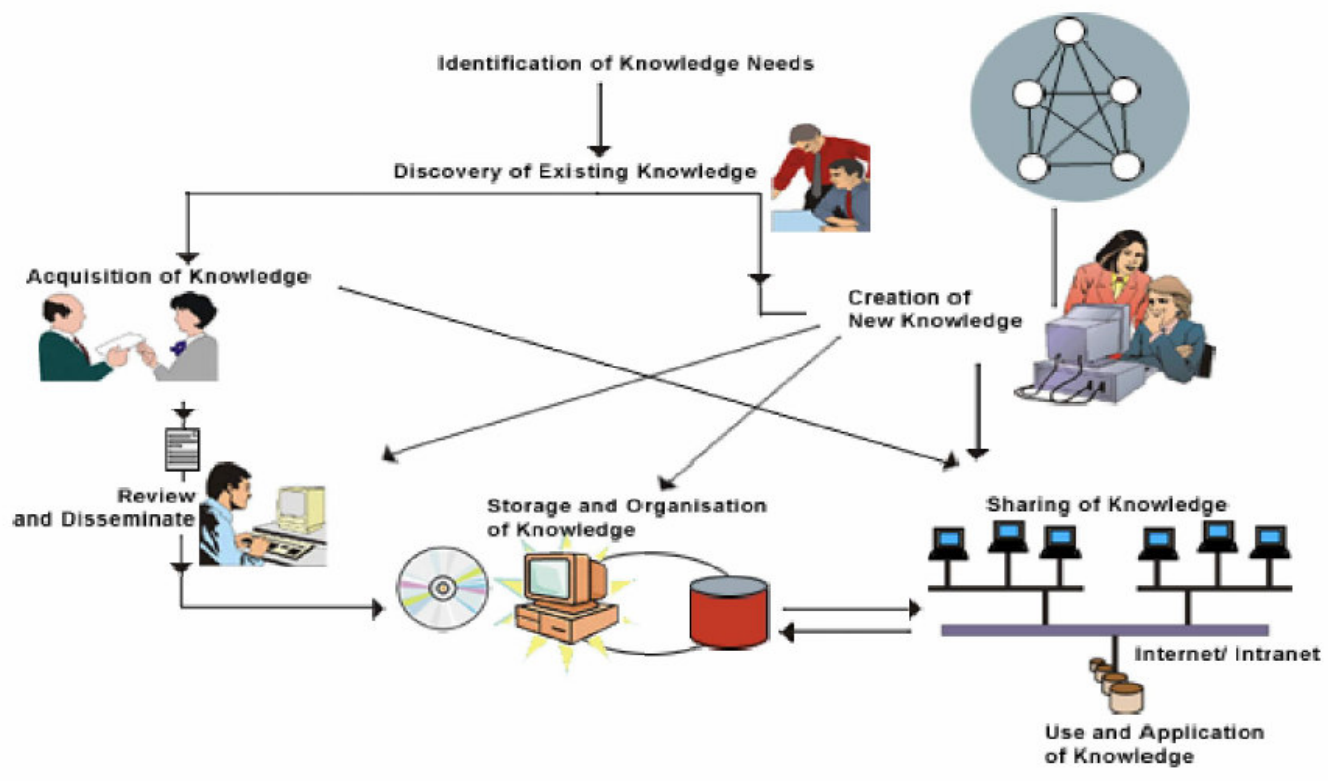

Figure 2: Knowledge Management Process

(Source: ANGLER at http://www.angleritech.com)

ICICI Onesource offers the following services: High-end Transaction Processing; Investment Research and Operations Research; Financial Analytics; Lower-End Transaction Processing (Application, processing, account maintenance); Outbound calling (Direct marketing, Verification, Loyalty Programs); Inbound calls (Customer service, Order Capture, Lead generation, Interactive Services; Complaint Resolution; Early-stage collections; Third Party collections; and Remittances \& Billing.

ICICI Onesource has a current capacity of 4500 seats across eight best-in-class delivery centers, of which, seven are in India's leading metros Mumbai, Bangalore and Delhi and one in Amherst, New York. The corporation is ISO/ IEC 17799:2000 certified and the world's first BPO to achieve COPC-2000 CSP certification for back office processing. It is also the first Indian BPO to adopt the BS 7799 security standards. It has the following knowledge assets: 


\section{Knowledge Management: Why Do We Need It for Corporates}

a) Quality process: The corporation adapted international standards such as COPC and BS ISO/IEC 17799:2000 as quality goals and continuous Six Sigma process improvement.

b) Strong management team: ICICI has team with a proven track record in financial services, technology, process and change management; international and culturally diverse team based in the UK, the US and India; and independent and experienced board of directors.

c) Proven record of managing relationships: ICICI has successful joint ventures with leading international financial services companies and relationships with global financial corporations developed over the last 50 years of its existence.

d) Domain expertise and experience: ICICI has proven expertise in offering BPO services to leading Fortune 500 and FTSE 100 companies. IOneSource can leverage the expertise of its parent and group companies.

e) Business continuity: ICICI has multiple centers in multiple cities; interconnected \& redundant telecom network; and verified disaster recovery plans and processes.

There is widespread recognition among knowledge-intensive organisations of the need to leverage their knowledge assets effectively. This is however tempered by the realization that the path to achieving this involves significant change - in terms of process, mindset and culture - within the organisation. It is unlikely that this change can be achieved in one giant leap with a planned KM initiative is thus desirable. As the primary objective of any corporate KM program is to support the achievement of strategic business objectives, the KM at ICICI OneSource enables achievement of business results through:

a) providing a framework which an organisation can use its KM Assets effectively. Knowledge is stored in various departments / sites respectively.

b) a mechanism to focus, and help prioritize, efforts to raise the level of business growth.

c) sharing of relevant Best-Practices, Case studies, lessons-learned from various training programs, etc. from both internal and external sources in areas that are relevant to the business.

d) implementation of appropriate IT tools to support knowledge-sharing \& collaboration both within the enterprise and in the extended enterprise - 


\section{Bhojaraju G.}

including customers, partners and suppliers - to help the organisation make better decisions.

e) formation of Communities of Practice (COP) by experts in each of the critical knowledge-capabilities identified earlier.

f) well-defined processes for knowledge-sharing (contribution) and knowledge-reuse (implementation)

g) corporate learning programs are conducted to enhance the ability of the staff.

h) conversion of individual knowledge into corporate knowledge assets to the maximum extent possible

i) facilitating the above through technology that supports knowledge-sharing $\&$ collaboration, pro-active "pushing" of relevant content to the right people who would act on them, formal and informal Knowledge-Sharing events such as seminars, discussion groups, etc., and encouraging the formation of "Communities of Interest" around Knowledge Competencies that are vital to the business.

While the KM initiative has been developed keeping the ICICI OneSource context and $\mathrm{KM}$ goals in mind, it is sufficiently generic to be used in any organisation which considers knowledge leverage as a significant determinant of success. What makes KM challenging is the fact that in many of today's dynamic organisations, the above-mentioned steps are not a one-time activity, but need to be re-validated on a continuous basis. Such organisations need to be pro-active to anticipate customer or market requirements and act on these ahead of competitors. Effective $\mathrm{KM}$ will enable the organisation to become nimble and make well-informed business decisions. A key-objective of KM is to ensure that the right knowledge is available with the right person at the right time in a "consistent and systematic" manner to enable timely decision-making.

An organisation's Knowledge Map must be a living organism that gets updated continuously as Knowledge Gaps are filled, new Subject Matter Experts are identified or new Knowledge Capabilities are defined. All contents are mapped planned in such a way that it will make a fine collaboration among teams working in various projects.

KM initiative has taken a good shape at ICICI OneSource with all issues, challenges as discussed above. It has included people, processes and technology in its purview. KM portal (Figure 3) has been deployed to its users on the Intranet 
Knowledge Management: Why Do We Need It for Corporates

where all the users of ICICI OneSource across worldwide centers including India are accessing and making use of the knowledge effectively in their day-to-day activities. SharePoint Portal Server 2003 serves as KM Tool and is designed to support the creation and maintenance of knowledge management solutions. Using the product, the organisation created a corporate portal that contains key information that all employees can access. The portal "sits on top" of the team portals built in Windows SharePoint Services. This allows users, with the proper access rights, to search for information in the corporate portal and in all team portals. In addition, users can post their expertise on personal portal pages. This allows others within the organisation to find experts quickly and easily. The main KM Sections in the portal are as follows: Best Practices, KM Cyberary, Training Feedback, Seminars, workshops, and conferences feedback, Staff publications, Experts Discussions, Minutes of Meetings, and Bulletin Boards, E-mails and Chat. The KM Cyberary section (Figure 4) provides gives links to various useful eresources Presentations, AV Materials, Reports, e-Resources Links and directory on Knowledge Management, Technology, BPO, and Call Centres.

$\mathrm{KM}$ encompasses every individual in the company - cutting across departments, functions, and business units. A robust Knowledge Management System (KMS) is indispensable to extract the useful knowledge and it is only logical that the system includes in its purview all the pertinent faces - the leadership, middle tier and the junior segment. Top management's ownership for the movement should be visible to the other tiers across various stages of KM deployment in order to propel the initiative. Being able to leverage one-another's knowledge should be seen as strength for the company. One aspect that knowledge evangelists would concur is the fulfillment of psychic needs as a result of knowledge sharing. Apart from the recognition one gets, the pure joy of sharing knowledge acts as a strong intrinsic motivator. With knowledge comes empowerment and by having a successful KM practice going, a company would be able empower every employee by the knowledge of every other employee. 
Bhojaraju G.

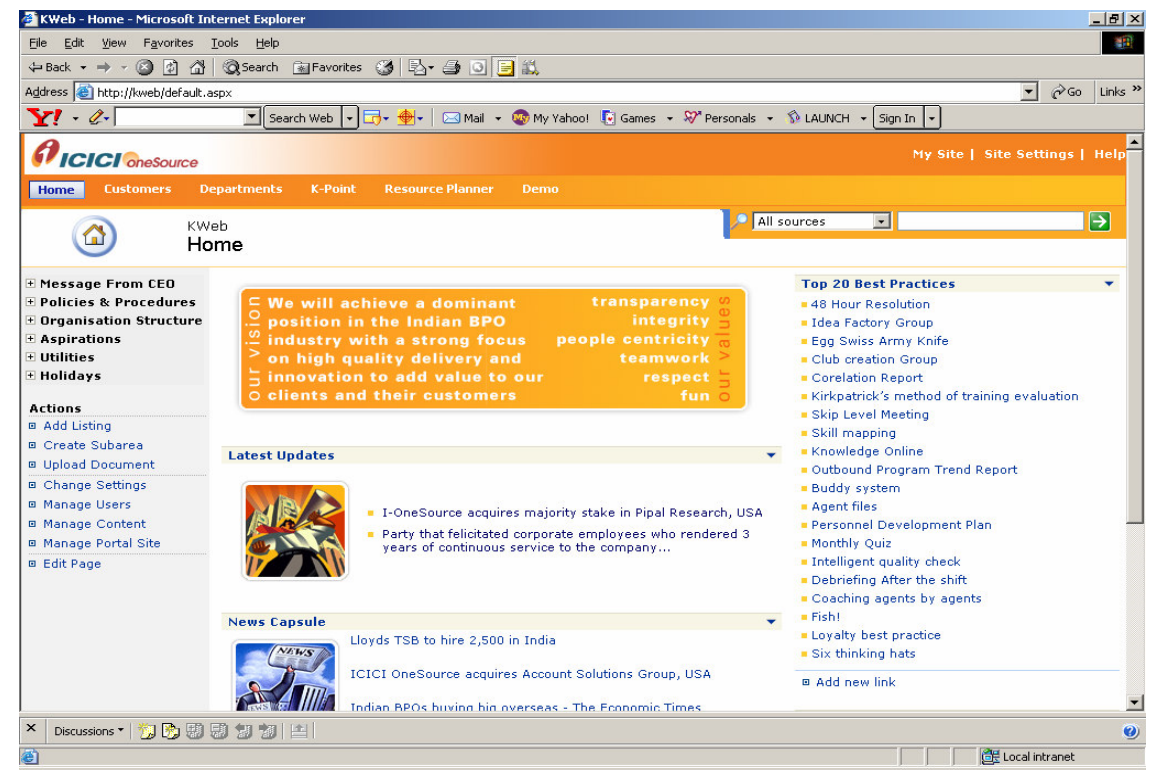

Figure 3: Knowledge Management Portal (http://www.icicionesource.com)

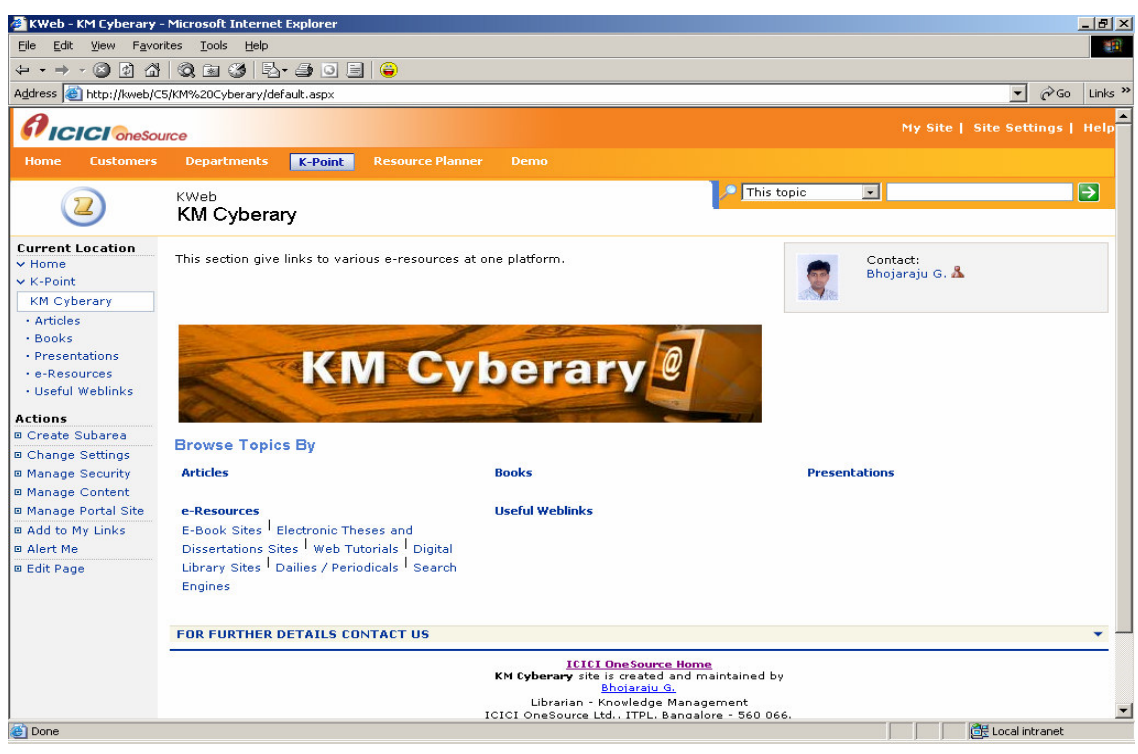

Figure 4: KM Cyberary Homepage 
Knowledge Management: Why Do We Need It for Corporates

\section{CONCLUSION AND IMPLICATION FOR POLICY AND FUTURE PRACTICES}

In today's technology world, the role of librarians or information professionals is deviating from traditional libraries' librarian role to virtual or digital libraries' Software Librarians, Cyberarians, Configuration Librarian or Librarian-Knowledge Management. Information Professionals are becoming Webmaster and Intranet Coordinators, combining technical expertise with information management ability.

Knowledge Management is an organisational approach that is not easily implemented. On one hand, knowledge-sharing activities depend on the voluntary participation of employees. Therefore, management should be sensitive to the knowledge activities that are already going on within the company and seek mean to support them. On the other hand, management needs to implement some organisational change in order to change the corporate culture. Employees can have all sorts of reasons for not joining in and employees who see benefit in KM have a hard time changing the corporate culture accordingly. Some objectives of workers can seem quite practical like, 'I don't have time for that, I need to meet my deadline'. Others could even be outright selfish, as they shield off their knowledge from potential competitors. A knowledge sharing culture can only found futile ground if top management supports it, showing benefits, incentives and rewards to those who do. Therefore KM cannot be just another project on the side; it requires structural change in the policy of the company.

KM requires a holistic and multidisciplinary approach to management processes and an understanding of the dimensions of knowledge work. KM should be the evolution of good management practices sensibly and purposively applied. KM presents a major shift in focus regarding the development and use of knowledge and information in increasing the effectiveness of any organisation.

It is true that knowledge capture of all tasks is possible, but one has to see the return of investment (ROI) point of view. It is very important to set the objectives for the system that one need to create. Based on the business goals, one can prioritize and identify critical information that can change organisational performance. The priorities might change based on changed business goals over time. The system has to be designed to cater to these changes. Therefore, it is important to think hard and plan for the long term, with short and long term objectives in mind. Instead of going directly to the search engine, one needs to 


\section{Bhojaraju G.}

look at the system in a holistic manner. For any KMS, the business objectives need to be set to ensure clarity of direction, else one can get lost in a forest of information.

\section{REFERENCES}

Abdul Kalam, A.P.J. 2004. Digital Library and its multidimensions. President of India's speech at the "Inauguration of International Conference on Digital Libraries (ICDL) - 2004, New Delhi : Feb 24, 2004. Available at: http://www.presidentofindia.nic.in/scripts/sllatest1.jsp?id=282

ANGLER. 2003. Full Service Provider (FSP) of Web, E-business and Multimedia. Available at http://www.angleritech.com

Broadbent, M (1997): The emerging phenomenon of knowledge management : The Australian Library Journal, Feb 6-24.

EIU.com. 2005. Know how: Managing knowledge for competitive advantage: Available at: http://www.eiu.com/KnowHow

GartnerGroup. 2005. Available at: http://www.gartner.com

ICICI OneSource. 2005. India's leading Business Process Outsourcing service provider. Available at http://www.icicionesource.com

Marr, Bernard. 2003. Consider the culture when benchmarking KM Processes. KM Review Vol. 6, no.5: 6-7.

Shadbolt, Nigel and O'Hara, Kieron. 2003. AKTuality : An overview of the Aims, Ambitions and Assumptions of the Advanced Knowledge Technologies Interdisciplinary Research Collaboration. Available at: http://www.aktors.org/ publications/selected-papers/01.pdf 